\title{
Target Ship Identification Algorithm Based on Comprehensive Correlation Discriminant and Information Entropy
}

\author{
Zhaoguo Shu \\ Shandong Institute of Space Electronic Technology Yantai, Shandong Province, China \\ Email: shuzhaoguo@163.com
}

How to cite this paper: Shu, Z.G. (2020) Target Ship Identification Algorithm Based on Comprehensive Correlation Discriminant and Information Entropy. Journal of Computer and Communications, 8, 61-71. https://doi.org/10.4236/jcc.2020.83007

Received: December 31, 2019

Accepted: March 6, 2020

Published: March 9, 2020

Copyright $\odot 2020$ by author(s) and Scientific Research Publishing Inc. This work is licensed under the Creative Commons Attribution International License (CC BY 4.0).

http://creativecommons.org/licenses/by/4.0/

\begin{abstract}
Ship type identification is an important part of electronic reconnaissance. However, in the existing methods, such as statistical-based methods and fuzzy-mathematics-based methods, the information acquired by the passive sensor is not fully utilized, and there is a certain ambiguity in the assignment relationship of the emitters-ship. They can't conclude the accurate and reliable assignment relationship of the emitters-ship. Therefore, this paper proposes a comprehensive correlation discriminant method to obtain a more reliable and comprehensive emitters-ship assignment, and then uses information entropy method to identify the type of the target ship on the basis of this association and assign the credibility. The simulation results show that this algorithm can effectively solve the problem of target ship type identification using the information of multi-passive sensors.
\end{abstract}

\section{Keywords}

Multi-Passive Sensor, Information Entropy, Target Ship Identification, Association Identification

\section{Introduction}

In modern warfare, electronic intelligence reconnaissance has always been the focus of research. Ocean surveillance mainly uses electronic reconnaissance satellites to detect and monitor sea targets. Electronic reconnaissance and surveillance satellites, such as the "Baiyun" series of electronic ocean surveillance satellites of the US Navy Ocean Surveillance system (NOSS), obtain the working parameters and location of the enemy radar system by detecting the electromagnetic radiation of the enemy radar system. Through the analysis of the working 
parameters and characteristic parameters of these radiation sources, the system, purpose and type of the target radiation sources can be obtained. Target platform recognition obtains the type and related information of the platform by analyzing the type and number of radiation sources equipped with the target platform [1]. Target platform identification is the basis for further analyzing the target-related weapon systems, working status and guidance methods of the platform, and understanding the characteristics of tactical application, activity rules and combat capability. It is also the key and main basis for high-level situation assessment and threat assessment. Under the background of marine electronic reconnaissance and surveillance, offshore target platform recognition, especially the type recognition of ship targets on the surface. There are a few researches on target platform recognition, and most of them focus on platform recognition based on emitter type recognition. The platform type of emitter is obtained by inference method based on the result of emitter type recognition. This method is difficult to achieve. In addition, the relationship between radar emitters and large ship platforms is generally not one-to-one correspondence. One type of radar radiation source may be loaded on many types of ship platforms, and there are often many types of radar radiation sources on one ship. Through the processing of the observed data of the target object, the output results will be used as the main basis for high-level situation assessment and threat assessment. Emitter recognition is to observe the characteristic parameters of radar and other emitters equipped with platform targets through electronic reconnaissance, and to infer their types by matching them with the characteristic parameters of known emitters in the database in some way. This is actually a process of template matching. Due to the complexity of the observation environment and the need for military secrecy, the data obtained by the sensor and the template in the database are incomplete, uncertain, especially fuzzy. The fuzzy set theory is used in target recognition, the characteristic parameters of the target are represented by membership function, and the uncertainty in recognition can be mathematically modeled effectively. In addition, fuzzy set theory provides a large number of fuzzy set operators, which makes the fuzzy matching algorithm have the advantages of simple operation and clear concept.

The result of electronic reconnaissance recognition is to use the recognition of the type of emitter equipped on the current platform and the allocation rules in the database of emitter-platform to convert the result of emitter recognition into a report based on the type of target platform. In fact, the conversion of electronic reconnaissance recognition report is based on the radiation source-platform allocation rule to realize reliability redistribution, so the allocation rule is the benchmark of report transformation. The quality of the rules affects the accuracy of the conversion of electronic reconnaissance reports and the recognition accuracy of the system. The current allocation rules are mainly based on the relationship between the technical parameters of the radiation source and the distribution platform, but this relationship is only relatively clear in the early wea- 
pons and equipment, with the development of radar emitter technology, one type of radar is often equipped on many types of platforms, so this relationship shows great fuzziness. The concept of allocating platform derived from the distribution of technical parameters of radar emitter is vague and uncertain. Data mining is a new technology to find and extract hidden information from historical transaction records in the database. The purpose of data mining is to help decision makers find potential knowledge and information between data. If there is a certain regularity between the values of two or more variables in the database, the regularity is called "association", which is a kind of important discoverable knowledge information in the database. By using the method of data mining [2], the intelligence obtained, the recognition experience of the operator and the historical recognition results stored in the recognition result database are deeply analyzed, and the interesting and potential radiation source-platform association rules are extracted. Complete the matching database, and use these association rules for the conversion of electronic reconnaissance reports to improve the accuracy of the conversion of electronic reconnaissance reports.

At present, there are four mainstream methods for target platform recognition:

1) Platform reasoning algorithm based on weighted fuzzy production rules

This reasoning algorithm is mainly divided into three steps: establishing rules, fuzzy matching and activating execution. The rules already exist in the knowledge base. On the one hand, the recognition result of the platform depends on the result of the recognition of the radiation source, on the other hand, it depends on whether the radiation source carried by the platform has a boot record. Both of them are very important for the identification of the platform.

By identifying the radar parameters equipped on the target platform, such as carrier frequency, pulse width, pulse amplitude, repetition frequency and so on, the type of target platform, such as the type of warship, is inferred by the method of fuzzy reasoning.

2) Target platform recognition algorithm based on information entropy

It is an urgent problem to be solved in radar countermeasure intelligence processing to study the potential association rules and knowledge between radar emitter and target platform, and how to transform the result of radar emitter recognition into platform discrimination more accurately and effectively. This method is a target platform association method based on information entropy to improve the accuracy and reliability of the target platform association method based on the results of radar emitter recognition, and uses information entropy to extract the feature information of the target platform assigned to the emitter and use this to associate and assign credibility to the target platform [3].

3) Recognition algorithm of emitter associated ship target platform based on evidence theory

The evidence theory method is a basic probability assignment method based on the personality differences of radiation sources carried on the warship-aircraft 
target platform. In the target fusion recognition system, because the information provided by each sensor is often incomplete, imprecise, fuzzy, has a certain degree of uncertainty and fuzziness, and may even contradict each other, therefore, in order to carry out target fusion recognition, we have to reason according to these uncertain information in order to achieve the purpose of obtaining the final identification of the target.

4) Identification method of target platform based on information entropy and DSmT

Using the identity judgment of the target provided by multiple sensors, especially the sensors of different systems and different uses, after certain processing, we can get a more accurate judgment, that is, target identity fusion. The combined identity report formed by target identity fusion is more accurate, specific and complete than that given by each sensor. DSmT well solves the contradiction that the DST method will produce counterintuitive conclusions in the case of high conflict of evidence [4].

The target of the maritime ship platform is in a moving state, and the tracking of the emitters on the ship by the electronic reconnaissance satellite can form the track of each emitter. In this paper, we propose a target ship recognition algorithm based on comprehensive correlation discrimination and information entropy. First, this method mainly uses the track correlation theory to correlate the tracks of each detected emitter, and determines the set of emitters belonging to the same target ship, and concludes the corresponding relationship between the emitters and the ship. And then we synthesize the emitters carried on each platform, and consider that the model of the target ship is identified using the information entropy-based target platform identification method using the results of the emitter model identification. The method is simple and reliable to implement, and the processing results can participate in target ship identification of heterogeneous multi-sensor data fusion, which has strong practicability.

\section{Target Ship Track Association}

\subsection{Problem Description}

Electronic Support Measurement (ESM) sensor is a passive sensor that can provide angle information and emitters attribute information (carrier frequency, pulse width, pulse repetition interval, etc.) in the target position [5]. These can obtain higher accuracy track correlation results, and provide a reliable basis for subsequent target ship model identification.

Let $E_{1}=\left\{e_{1}, e_{2}, \cdots, e_{n}\right\}, E_{2}=\left\{e_{1}, e_{2}, \cdots, e_{m}\right\}$ be the track number sets of sensor 1 and sensor 2, respectively. The combined detection of targets by multiple passive sensors can effectively improve the positioning accuracy of the target, obtain the precise position information and attribute information of the target, and thereby obtain the radiation located on the same ship [6]. As shown in Figure 1, it shows the improvement of positioning accuracy between multi-sensor and single sensor. 


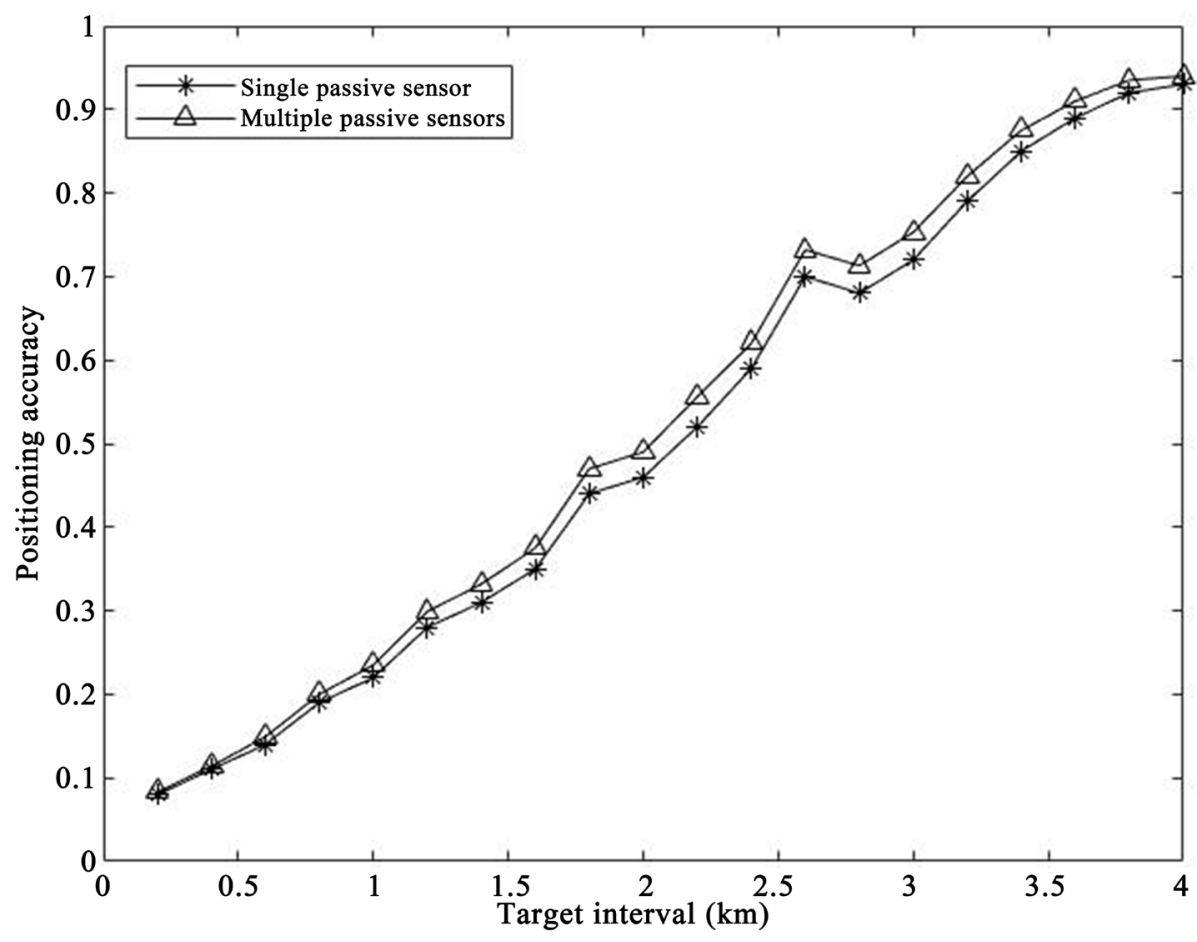

Figure 1. Comparison of positioning accuracy between multi-sensor and single sensor.

\subsection{Track Autocorrelation Correlation Discrimination}

For the correlation of the emitter tracks obtained by the same sensor, the combination of the detected azimuth, elevation and emitters attribute information should be used in the selection of the discriminant function, so as to improve the accuracy of the track auto-correlation [7].

1) Angle information correlation discriminant function

Let $\alpha_{i j}, \theta_{i j}$ be the $i^{\text {th }}$ azimuth and pitch measurements of the $f^{\text {th }}$ track; and $\alpha_{i k}, \theta_{i k}$ are the $i^{\text {th }}$ azimuth and pitch-angle measurements of the $k^{\text {th }}$ track. $\alpha_{i}=\alpha_{i j}-\alpha_{i k}$ represents the $f^{\text {th }}$ track's The difference between the $i^{\text {th }}$ azimuth and the $i^{\text {th }}$ azimuth of the $k^{\text {th }}$ track; $\theta_{i}=\theta_{i j}-\theta_{i k}$ represents the difference between the $i^{\text {th }}$ elevation of the $f^{\text {th }}$ track and the $i^{\text {th }}$ elevation of the $k^{\text {th }}$ track. When track correlation is performed, the measurement errors of elevation and azimuth are the same, and follow a normal distribution with zero mean constant variance. The variance is expressed as $\sigma$, and the construction statistics are

$$
\begin{aligned}
& \Delta \alpha=\sum_{i=1}^{N}\left(\frac{\alpha_{i j}-\alpha_{i k}}{\sqrt{2} \sigma}\right)^{2} \\
& \Delta \theta=\sum_{i=1}^{N}\left(\frac{\theta_{i j}-\theta_{i k}}{\sqrt{2} \sigma}\right)^{2}
\end{aligned}
$$

Obviously, when the trajectory of the $f^{\text {th }}$ emitters and the trajectory of the $k^{\text {th }}$ emitters are from the same target platform, they conform to the standard normal distribution with a mean of 0 and a variance of 1 , that is $\Delta \alpha \sim N(0,1)$, 
$\Delta \theta \sim N(0,1)$, and satisfy the properties of general similarity measurement. Therefore, the Equations (1) and (2) are selected as the correlation functions of the azimuth and elevation of the track.

2) Attribute correlation discriminant function

Let other parameters of the emitters (such as carrier frequency, pulse width, pulse repetition interval, etc.) be $p_{i}$, and their errors are independent of each other, and obey the Gaussian distribution with mean value 0 and variance $\sigma_{p}$, then $\Delta p_{i}=\frac{p_{i j}-p_{i k}}{\sqrt{\sigma_{p_{i j}}^{2}+\sigma_{p_{i k}}^{2}}}$ based on the variables of each attribute information obeys the standard normal distribution. If the attribute information has $\mathrm{M}$ dimensions, then let

$$
\begin{gathered}
c_{p}=\sum_{i=1}^{M} \Delta p_{i}, \text { then } \\
c_{p}=\frac{1}{\sqrt{2 \pi}} \exp \left(-\frac{1}{2} \Delta p_{i}^{2}\right)^{2}
\end{gathered}
$$

In the equation, $p_{i j}$ represents the $i^{\text {th }}$ attribute information of the $f^{\text {th }}$ track; $p_{i k}$ represents the $i^{\text {th }}$ attribute information of the $k^{\text {th }}$ track. Equation (3) satisfies the properties of general similarity measurement, so Equation (3) is selected as the attribute correlation discriminant function of the track.

3) Comprehensive correlation discriminant function

The expressions obtained from the Equations (1) to (3) all obey the normal distribution and are independent of each other. Combining them, we can get

$$
L_{i}=\Delta \theta^{2}+\sum_{i=1}^{M} \Delta p_{i}^{2}+\Delta \alpha^{2}
$$

The above equation obeys the distribution $\chi^{2}(n+1)$, and its dimensions are one-dimensional in elevation and one-dimensional in azimuth, multi-dimensional configuration of parameter information.

\subsection{Track-Related Decision Rules}

Let $\lambda$ be the comprehensive judgment value, in order to give the similarity judgment between the trajectory $j\left(j \subset E_{1}\right)$ and the trajectory $k\left(k \subset E_{1}\right)$, sort the similarity from large to small, and adopt the principle of maximum discriminant identification. That is, if $\lambda^{*}=\max \left(\lambda_{i}\right)$, and $\lambda^{*}>\varepsilon$, the track $j\left(j \subset E_{1}\right)$ at time $t$ is associated with the track $k\left(k \subset E_{1}\right)$, which $\varepsilon(0.5 \leq \varepsilon<1)$ is the threshold value, which can be determined through simulation experiments [8].

In order to control the completion and termination of track correlation inspection and improve the speed of the algorithm, we introduced the quality of track correlation $m_{j k}(t)$. Correlation quality is a measure of the history of track associations [9]. The value of the association quality reflects the reliability of correct associations. If it is judged that the track $j\left(j \subset E_{1}\right)$ and the track $k\left(k \subset E_{1}\right)$ are related pairs at time $t$, then 


$$
m_{j k}(t)=m_{j k}(t-1)+1
$$

otherwise

$$
m_{j k}(t)=m_{j k}(t-1)-1
$$

In the equation, $m_{j k}(0)=0$. When $m_{j k} \geq 8$, we think that track $j\left(j \subset E_{1}\right)$ and track $k\left(k \subset E_{1}\right)$ are fixedly associated, and it is no longer necessary to check them in the future, and directly enter the track fusion stage.

Then, in the same way, the processing results obtained by different sensors are associated and identified, and the result of track fusion can be obtained, and the types of partial emitters located on the same ship can be known. Then we use the method based on information entropy to identify the ship.

See Algorithm 1 for details of the passive track association algorithm based on comprehensive correlation discrimination.

\section{Identification of Target Ship Model Based on Information Entropy}

After comprehensive relevant judgments, a part of the emitters set located on the same ship is obtained, and the assignment relationship between the emitters and the ship is obtained [10]. There are a variety of $u$ emitters types and a variety of $V$ target ship types, and the assignment relationship characteristic matrix $\mathrm{A}$ is constructed as follows:

$$
A=\left(\begin{array}{ccc}
a_{11} & \cdots & a_{1 v} \\
\vdots & \ddots & \vdots \\
a_{u 1} & \cdots & a_{u v}
\end{array}\right)
$$

In the equation, $a_{i j}$ represents the assignment relationship between the $\mathrm{i}$-type emitter and the $\mathrm{j}$-type ship. If the $\mathrm{j}$-type ship is equipped with the $\mathrm{i}$-type emitter, then $a_{i j}=1$, otherwise $a_{i j}=0$.

Algorithm 1. Getting the assignment relationship between the emitters and the ship.

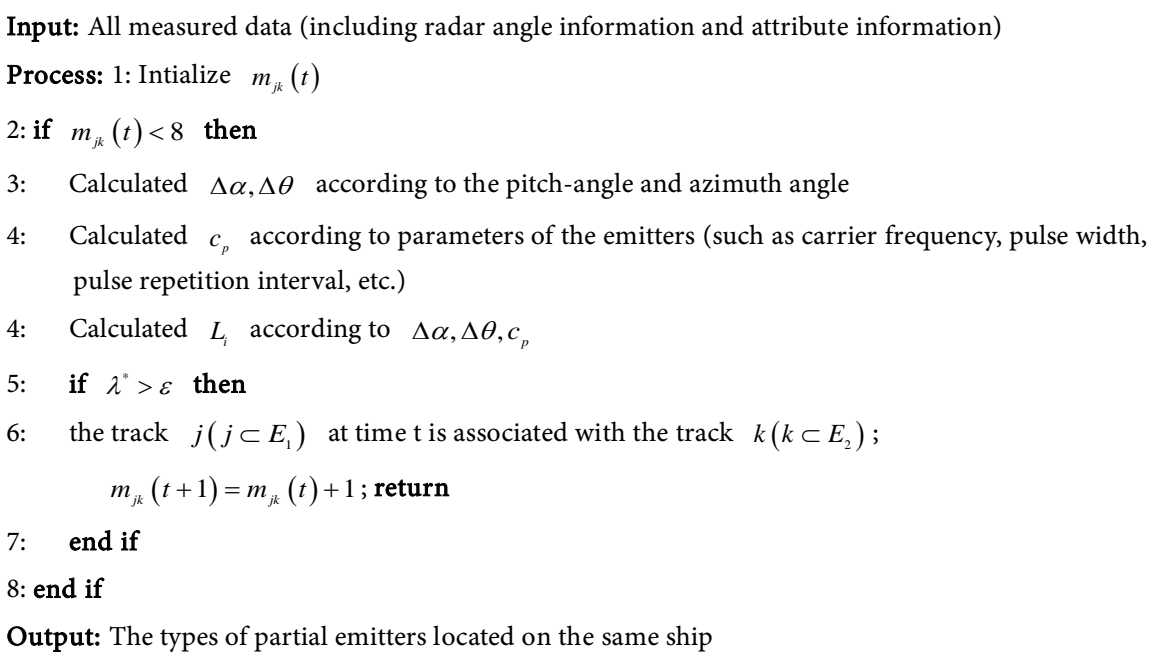


For the i-type emitter, the information entropy value is:

$$
H_{i}=-k \sum_{j=1}^{v} \gamma_{i j}
$$

among them $i=1,2, \cdots, u, v>1, k=\frac{1}{\ln (v)}$.

$$
\gamma_{i j}=\left\{\begin{array}{l}
0, f_{i j}=0 \\
f_{i j} \ln \left(f_{i j}\right), f_{i j} \neq 0
\end{array}, f_{i j}=a_{i j} / \sum_{j=1}^{v} a_{i j}\right.
$$

The information entropy represents the amount of information that the type emitter can appear when identifying the target platform. Because the size of information entropy is inversely related to the amount of information it contains, it is not intuitive and easy to use for identification decisions, so it can be transformed into entropy weights:

$$
\varphi_{i}=\frac{1-H_{i}}{u-\sum_{i=1}^{u} H_{i}}
$$

Suppose that in a certain reconnaissance, the emitters on a certain platform are identified through electronic reconnaissance. Based on the existing knowledge, this emitter may be equipped with different types of $v$ target ships. Therefore, a decision matrix $\mathrm{R}$ can be constructed as:

$$
R=\left[\begin{array}{cccc}
\varphi_{1} r_{11} & \varphi_{1} r_{12} & \cdots & \varphi_{1} r_{1 v} \\
\varphi_{2} r_{21} & \varphi_{2} r_{22} & \cdots & \varphi_{2} r_{2 v} \\
\vdots & \vdots & \ddots & \vdots \\
\varphi_{u} r_{u 1} & \varphi_{u} r_{u 2} & \cdots & \varphi_{u} r_{u v}
\end{array}\right]
$$

The $r_{i j}$ assignment rules of are as follows:

If the $\mathrm{j}$-type ship is equipped with an i-type emitter $r_{i j}=\mu_{i j}\left(0 \leq \mu_{i j}<1\right), \mu_{i j}$ is the credibility of the identification of the emitters and also indicates the support of the i-type emitters for the j-type ship; if the j-type target is not equipped with a i-type emitter, then $r_{i j}=0$.

Calculate the comprehensive decision value according to the decision matrix:

$$
z_{j}=\sum_{i=1}^{u} \varphi_{i} r_{i j}
$$

Therefore, the comprehensive decision value can be used to discriminate the target platform and achieve credibility conversion. Proceed as follows:

1) Target platform identification

The calculation selects the target ship with the largest comprehensive decision value as the recognition result. If the comprehensive attribute value corresponding to the target ship $p$ is the largest, the target ship to be identified is classified as $p$, which is expressed as:

$$
z_{p}=\max \left(z_{j}\right) \quad(j=1,2, \cdots, v) .
$$

2) Credibility assignment 
For the identified target ship, the recognition credibility of the emitter can be converted into the recognition credibility of the target ship according to the entropy weight. Let there be $\mathrm{M}$ emitter recognition results, where the recognition credibility of the emitter is $r_{i}$, and the corresponding entropy weight is $\varphi_{i}$. Then the credibility of the target ship is:

$$
m_{T}=\sum_{i=1}^{M}\left(\varphi_{i} r_{i} / \sum_{i=1}^{M} \varphi_{i}\right)
$$

\section{Case Analysis}

To evaluate the performance of our proposed method, we conducted experiments on the following examples. After calculation and statistics of the above algorithm, the assignment relationship and entropy weight of type 6 emitters and type 6 ships are obtained, as shown in Table 1 .

In a certain mission, the confidence level of the type 6 emitter identification (that is, the support for each ship) is shown in Table 2.

The comprehensive decision value of each ship calculated by using equation (12) is shown in Figure 2. According to the maximum comprehensive decision value, the recognition result is ship $\mathrm{D}$, and its recognition credibility is $m_{T}=\sum_{i=1}^{6}\left(\varphi_{i} r_{i} / \sum_{i=1}^{6} \varphi_{i}\right) \approx 0.8939$.

\section{Conclusion}

In this paper, we propose a target ship model recognition algorithm based on comprehensive correlation discrimination and information entropy. First, the track of each radiation source detected is correlated by using the comprehensive correlation discrimination method, and the radiation source set belonging to the

Table 1. Emitters and target ships entropy weight relationship.

\begin{tabular}{|c|c|c|c|c|c|c|}
\hline Ship & Ship A & Ship B & Ship C & Ship D & Ship E & Ship F \\
\hline Emitter 1 & 0.016 & 0 & 0.016 & 0 & 0.016 & 0 \\
\hline Emitter 2 & 0 & 0 & 0.0288 & 0.0288 & 0 & 0 \\
\hline Emitter 3 & 0.016 & 0.016 & 0 & 0 & 0.016 & 0 \\
\hline Emitter 4 & 0 & 0.016 & 0.016 & 0.016 & 0 & 0 \\
\hline Emitter 5 & 0 & 0 & 0 & 0.0288 & 0.0288 & 0 \\
\hline Emitter 6 & 0.007 & 0.007 & 0 & 0.007 & 0 & 0.007 \\
\hline
\end{tabular}

Table 2. Emitter confidence.

\begin{tabular}{ccccccc}
\hline Emitter & Emitter 1 & Emitter 2 & Emitter 3 & Emitter 4 & Emitter 5 & Emitter 6 \\
\hline Recognition Credibility & 0.7 & 0.9 & 0.8 & 0.95 & 0.86 & 0.88 \\
\hline
\end{tabular}




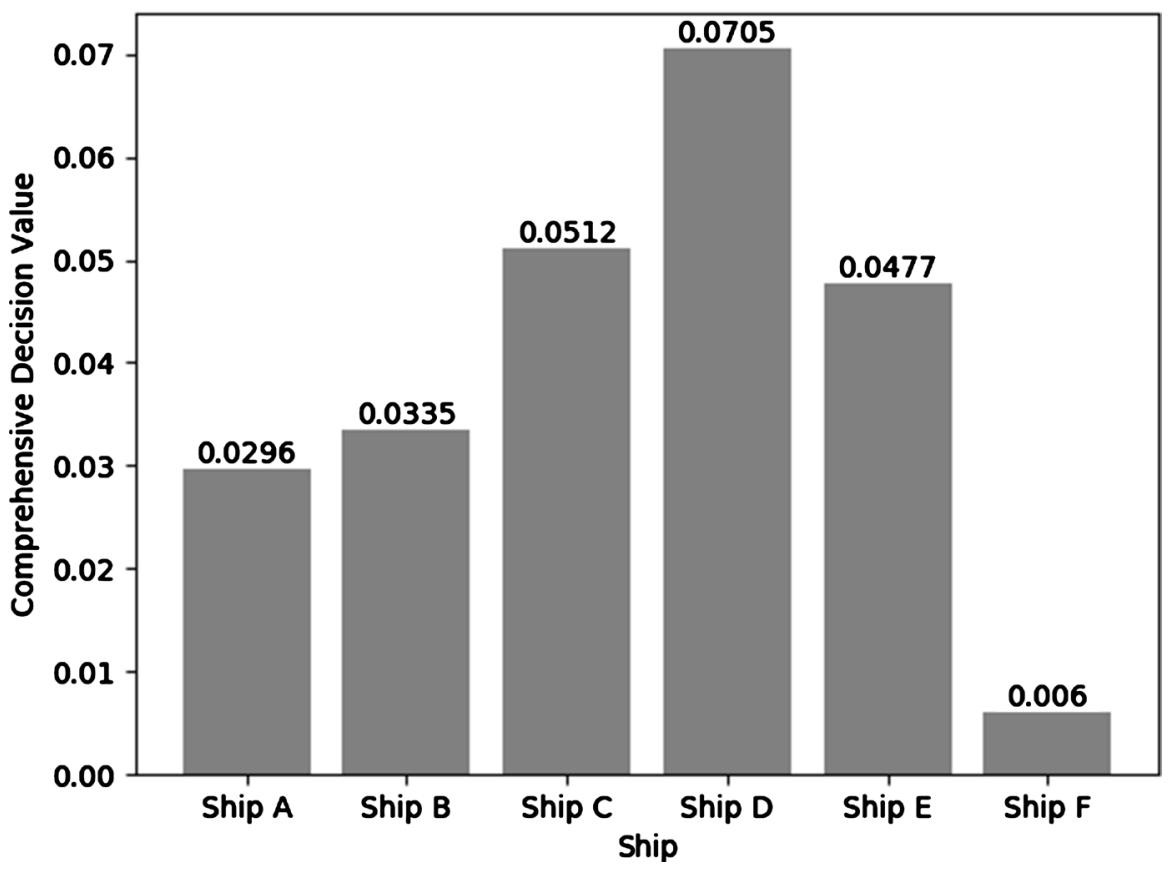

Figure 2. Comprehensive decision value.

same target ship is determined, and the corresponding relationship between the radiation source and the platform is obtained. Then, comprehensive consideration is given to the radiation sources carried on each platform, and the type of the target ship is identified through the target platform identification method based on the information entropy using the radiation source model identification results. Compared with the existing methods, this paper makes full use of the information obtained by electronic reconnaissance technology, and improves the reliability of obtaining the set of radiation sources located on the same ship through comprehensive correlation discrimination. Furthermore, the type of the target ship is quickly determined by using the target platform recognition based on information entropy, which makes the whole process reliable, fast and efficient. After simulation experiments, this method can effectively complete the task of target recognition, and the recognition results can be further used in information fusion.

\section{Conflicts of Interest}

The author declares no conflicts of interest regarding the publication of this paper.

\section{References}

[1] Haviland, S., Correa, W., Poole, E., Garris, J. and Jones, T. (2018) Information Warfare at the Battle of Belleau Wood. Marine Corps Gazette, 102, 32-44.

[2] Maruyama, M., Flath, D., Minamikawa, K., Ohkita, K. and Zennyo, Y. (2015) Platform Selection by Software Developers: Theory and Evidence. Journal of the Japanese and International Economies, 38, 282-303. 
https://doi.org/10.1016/j.jjie.2015.10.003

[3] Gao, J.M., Gong, L.L. and Lu, T. (2013) Target Platform Identification Method Based on Information Entropy. Computer Application and Software, 30, 224-227.

[4] Deng, Y.F. and Zhang, H.B. (2015) Target Platform Identification Based on the United Method of Comentropy and DSmT. Shipboard Electronic Countermeasure, 38, 15-18.

[5] Liu, Z., Feng, X.X., Zhou, H. and Liu, Y.L. (2012) Algorithm of Passive Track Correlation Based on Synthesis Correlation Discrimination. Electronic Optics and Control, 19, 20-24.

[6] Zhu, B.H., Feng, X.X., Lu, C.G. and Wang, B. (2011) A Track Association Algorithm of Heterogeneous Sensors Based on Gray Theory by Using Information Synthetically. Electronic Optics and Control, 18, 22-25.

[7] Dong, H.B., Wang, G., Liu, J.M. and Zhang, C.M (2010) Research on Track Correlation of Multi-Sensor in Airborne. Transducer and Microsystem Technologies, 29, 12-14.

[8] Dai, J.J. and Li, X.M. (2013) Threat Evaluation of Air Strike Targets for Warship Formation Networked Air-Defense Operation. Journal of Systems Engineering and Electronics, 35, 532-538.

[9] Yan, K.Y., Wang, J.G. and Bai, R.H. (2010) Track Association Algorithm with Multi-Feature Based on Distributed Multi-Target Passive Tracking System. Information and Electronic Engineering, 8, 550-554.

[10] Yang, Y.Z., Zhou, Z.L. and Liu, H.Q. (2018) Threat Evaluation Method of Air Target Based on Information Entropy and Rough Set. Journal of Beijing University of Aeronautics and Astronautics, 44, 2071-2077. 